\title{
Development and the domestic chicken
}

\author{
SCIENTIFIC NAME \\ Gallus gallus domesticus \\ TAXONOMY \\ PHYLUM: Chordata \\ CLASS: Aves \\ ORDER: Galliformes \\ FAMILY: Phasianidae
}

\section{Physical description}

The domestic chicken's distinguishing characteristics include the comb atop its head (hence its Latin name; gallus in Latin means comb) and two wattles beneath its beak, features that are more prominent in the male. In research, the most commonly used breeds of chicken are the White Leghorn, which sport white feathers, yellow feet and yellow beaks, and the Rhode Island Red, which have reddish or dark brown feathers, red-orange eyes, yellow feet and reddish-brown beaks. These breeds are preferred for research because they are prolific egg-layers (around 250 to 300 per year). Like other birds, they have hollow bones, making their bodies lighter to facilitate flying. Also lightening the load are 12 air sacs in their bodies, which function as part of the respiratory system. Despite these adaptations, domestic chickens have mostly lost their ability to fly as they have been bred to encourage larger body mass for meat production.

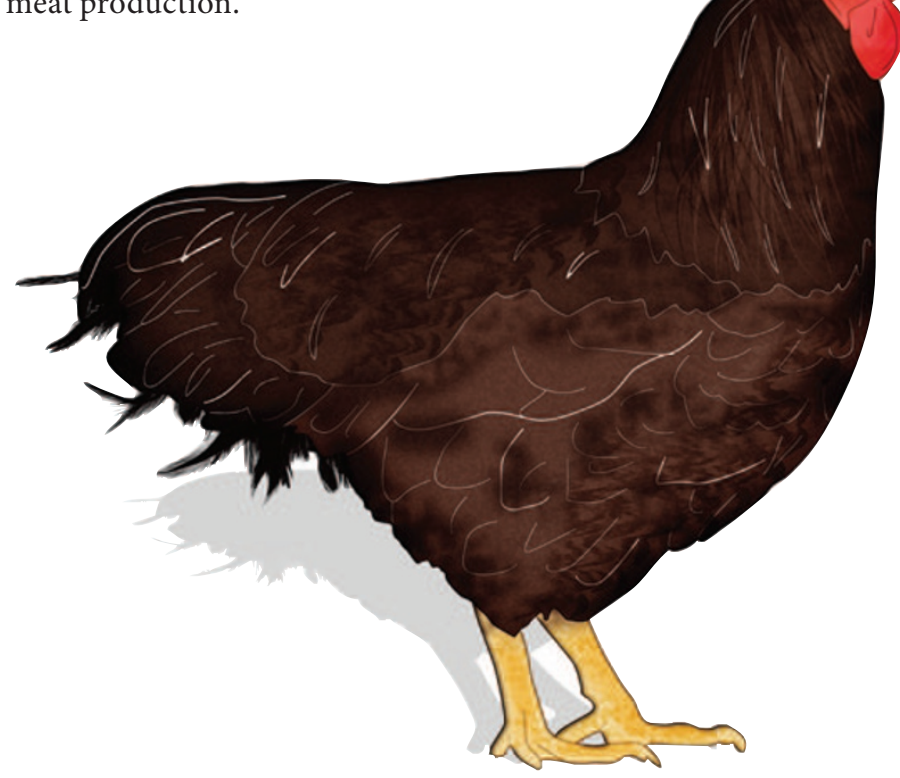

\section{Genetic background}

The chicken genome was first sequenced in March 2004 by the International Chicken Genome Sequencing Consortium. There is currently a huge funding push to support annotation of the chicken genome to support the many research areas utilizing this model animal. So far, many similarities have been found with the human genome, enhancing its usefulness in genetic studies. The fairly small genome size ( $\sim \mathrm{Gb})$ makes the species ideal for research using techniques that reveal the genetic basis for rapid evolutionary adaptations ${ }^{1}$.

\section{Research résumé}

The chicken is an appealing comparative model to study molecular mechanisms leading to testis and ovary formation ${ }^{2}$. Gonad development is similar in birds and mammals, sharing features such as asymmetry of the female reproductive system and the sensitivity of gonad differentiation to estrogens ${ }^{2}$. Additionally, the chicken is the only model species in which the female is heterogametic and there is a high degree of differentiation between two sex chromosomes ${ }^{2}$.

Chickens are also commonly used in the study of retinal diseases. The rdd (retinal dysplasia and degeneration) chicken was developed as a model of retinal degeneration in humans ${ }^{3}$. Leber congenital amaurosis (LCA), a genetically heterogenous group of retinal diseases that cause congenital blindness in infants and children, has also been modeled in the chicken. The GUCY $1^{\star} \mathrm{B}$ chicken carries a null mutation of the gene $G C 1$ (guanylate cyclase-1) that disrupts the synthesis of cyclic GMP in photoreceptor cells, a messenger protein required for function of these cells, and causes blindness at hatching, a phenotype that closely matches that observed in humans with LCA type 1 (ref. 4). The GUCY1*B chicken serves as an animal model for the study of LCA1 pathology and potential human treatments, including therapeutic applications for GC1 transgenes. Lentivirus-based therapies with such transgenes have restored electroretinographic responses and slowed retinal degeneration in the chicken model ${ }^{4}$, providing hope for potential treatments for humans with the disease.

\section{FOR MORE INFORMATION}

http://www.nih.gov/science/models/gallus/

1. Rubin, C. et al. Whole-genome resequencing reveals loci under selection during chicken domestication. Nature 464, 587-591 (2010).

2. Carre, G., Couty, I., Hannequet-Antier, C. \& Govoroun, M.S. Gene expression profiling reveals new potential players of gonad differentiation in the chicken embryo. PLoS One 6, e23959 (2011).

3. Finnegan, S. et al. Proteomic profiling of the retinal dysplasia and degeneration chick retina. Mol. Vision 16, 7-17 (2010).

4. Williams, M.L. et al. Lentiviral expression of retinal guanylate cyclase-1 (RetGC1) restores vision in an avian model of childhood blindness. PLoS Med. 3, e201 (2006). 